\title{
Management of patients with colorectal cancer in COVID-19 pandemic hospital
}

\author{
Catal O, Ozer B, Sit M \\ Abant Izzet Baysal University Hospital, Department of General Surgery, Bolu, Turkey. \\ otuzogur@gmail.com
}

\begin{abstract}
INTRODUCTION: During the COVID-19 pandemic, both the channelling of manpower into the COVID-19 services and efforts to limit viral spreading of the disease have led to rescheduling and prioritizing oncological cases that are considered as cases of emergency and any postponement of the cases can lead to health problems. In this study, we aimed to show that the surgical treatment of COVID-19 (-) colorectal cancer patients can be performed in a hospital which was declared as a pandemic hospital.

METHOD: In our study, we included patients who underwent surgery for colorectal cancer in the general surgery clinic between the dates 03.20.2020 and 05.20.2020, when the hospital was declared COVID-19 pandemic hospital. Emergency colorectal cancer surgeries such as ileus and perforation were excluded from the study.

RESULTS: While in the status of pandemic hospital, 80 COVID-19 (+) and 563 COVID-19 (+) suspicious patients were treated. Between 10 March 2020 and 15 May 2020, 12 colorectal cancer patients were operated in the General Surgery Clinic of BAIBU Faculty of Medicine, as well.

CONCLUSION: We think that with our study we have shown that during the COVID-19 pandemic, the surgical treatment can be performed for patients with colorectal cancer by eliminating COVID-19 suspicions (Tab. 1, Ref. 14). Text in PDF www.elis.sk KEY WORDS: COVID-19, pandemic, colorectal cancer.
\end{abstract}

\section{Introduction}

In December 2019, a new and highly contagious coronavirus infection outbreak was detected in Wuhan, China. Called SARSCoV-2 by the World Health Organization (WHO), the pathogen is responsible for a new pneumonia called Coronavirus Disease 2019 (COVID-19) that affects the lower respiratory tract (1). The most common symptoms were fever, cough, weakness and shortness of breath (2). Later, gastrointestinal system symptoms such as diarrhoea were identified (3). It has been found that COVID-19 is mainly transmitted from person to person, who are in close contact with each other, by airborne droplets (4). It was declared as COVID-19 pandemic by the World Health Organization on 11 March 2020 (5). The first COVID-19 case in Turkey was seen on March 10, 2020. The excessive increase of patients who were in need of intensive care caused an unprecedented crisis for health systems around the world. During the COVID-19 pandemic, all segments of the society has continued to be affected by existing diseases, therefore; the COVID-19 outbreak put healthcare institutions in a density they could not overcome. During the COVID-19

Abant Izzet Baysal University Hospital, Department of General Surgery, Bolu, Turkey

Adress for correspondence: $\mathrm{O}$. Catal, MD, Abant Izzet Baysal University Hospital, Department of Surgery, Golkoy, 14280, Bolu, Turkey. Phone: +903742534656, Fax: +903742534615 pandemic, both the channelling of manpower into the COVID-19 services and efforts to limit viral spreading of the disease have led to rescheduling and prioritizing oncological cases that are considered as cases of emergency and any postponement of the cases can lead to health problems (6).

Although there have been guidelines published by general surgery associations and health institutions about the management of clinics and patients, there is a lack of evidence-based literature. At the beginning of March 2020, Centres for Disease Control and Prevention (CDC) recommended postponing/rescheduling the time of elective surgeries and performing outpatient treatment of inpatients if possible (4).

Colorectal cancer is the third most common cancer in the world. When it is evaluated according to gender, it is the third most common cancer in men after prostate and lung cancer and the second most common cancer in women after breast cancer. It is more common in men than in women. In colorectal cancers, the doubling time has been reported as an average of 130 days (7). The transition to the normalization process is not fully predictable, therefore; delaying the surgical treatment of patients, who underwent surgical curative procedures such as colorectal cancer during the COVID-19 pandemic, will negatively affect the patient's life span.

In this study, we wanted to showcase that the surgical treatment of COVID-19 (-) colorectal cancer patients can be performed by creating self-protective equipment and operating room conditions 
in accordance with the guidelines published by Bolu Abant Izzet Baysal University hospital, which was declared as a pandemic hospital by the Turkish Ministry of Health on 20.03.2020.

\section{Method}

In our study, we included the patients who underwent surgery for colorectal cancer in the general surgery clinic of Bolu Abant Izzet Baysal University Training and Research Hospital between the dates 20.03.2020 and 20.05.2020, when the hospital was declared COVID-19 pandemic hospital by the Republic of Turkey Ministry of Health. Emergency colorectal cancer surgeries such as ileus and perforation were excluded from the study. During the period of our clinic being used as pandemic hospital, the patients who applied for colorectal cancer surgery were primarily evaluated for possible COVID-19 infection. COVID-19 related symptoms and possible contacts with COVID-19 (+) patients were asked and questioned. The history of recent foreign trips was questioned on the basis of possible contacts with COVID-19 (+) carriers and patients. On physical examination of the patients, fever, saturation, and auscultation of the lungs were evaluated and noted. Then, CRP, lymphocyte, ferritin, d-dimer values of the patients were examined in the preoperative blood tests. In terms of colon cancer screening, abdominal and thorax CT scans were carried out and the results were evaluated for both metastasis and COVID-19 findings. COVID-19 (-) colorectal cancer patients were hospitalized in different services, in single isolated rooms and physically separated blocks from the patient services where follow-up and treatments were applied for COVID-19 (+) patients.

During the pandemic period, the locations of the operating rooms in the hospital were changed. The current operating room used by the general surgery clinic was designed and allocated for the COVID-19 (+) patients in need of surgery. The cardiovascular surgery operating rooms, which are located in different blocks and physically separated from the services where COVID-19 (+) patients are treated, were arranged to operate COVID-19 (-) patients. We performed surgical procedures for colorectal cancer patients in these operating rooms (the cardiovascular surgery operating rooms), as well.

\section{Results}

The first COVID-19 case in Turkey was seen on March 10, 2020. On 20 March 2020, Republic of Turkey Ministry of Health declared Bolu Abant Izzet Baysal University Medical Faculty Hospital as one of the pandemic hospitals in Turkey. As of that date, with the decision of the hospital management, the services and operating rooms for the hospitalization of COVID-19 $(+)$ patients were determined. It was decided to operate only patients with emergency cases and cancer patients; the surgery plans of elective and treatable patients were delayed and stopped.

Bolu Abant Izzet Baysal University Medical Faculty Hospital is a hospital with 340 beds. Between 20 March 2020 when BAIBU Medical Faculty Hospital was declared as a pandemic hospital and 01.06.2020 when it was removed from pandemic hospital status, 80 COVID-19 (+) and 563 COVID-19 (+) suspicious patients were followed-up and treated. Between 10 March 2020 and 15 May 2020, 12 colorectal cancer patients were operated in the General Surgery Clinic of BAIBU Faculty of Medicine, as well. The average age of the operated colorectal cancer patients is 63.66 (37-77). Demographic features including gender distributions, post-operative hospitalization times and post-operative complications of patients are shown in Table 1. The surgical planning has not been postponed since all of the rectal cancer patients had passed the mark of 8-10 weeks duration of time after neoadjuvant chemo-radiotherapy. According to the results of the triage examinations of colorectal cancer patients admitted to our clinic, all of the patients were concluded as COVID-19 (-). Open surgical technique was applied to all patients. Laparoscopic procedure has not been attempted/tried.

In the post-operative 30-day follow-up of the patients who were operated for colorectal cancer during the COVID-19 pandemic, no further development/problem of COVID-19 infection was detected. The patients were rechannelled to oncology outpatient clinics for adjuvant oncology treatment after the surgical follow-up time was completed.

\section{Discussion}

It has been deemed appropriate to perform surgeries for emergency diseases and for the diseases that decrease the patient's survival process when the elective operations and treatments are delayed/postponed during the COVID-19 pandemic (8). So, how to approach the patient who is about to enter the operating room during the COVID-19 pandemic? Studies related to this indicate that it is important to try preventing cross infec- 
tion before the patient enters the operating room. The first triage should be performed before admission of the patient to the operating room and the suspicion of COVID-19 should be evaluated. If there are no findings to be suspicious of COVID-19, the second triage should be carried out. In the second triage, the body temperature, the laboratory findings (especially lymphocyte count, ferritin level) and chest x-ray/tomography findings (especially the findings of ground glass opacity) should be evaluated and these findings should eliminate COVID-19 suspicions. If there is no doubt/suspicion of COVID-19, routine anaesthesia and surgery should be performed. However, if the patient has a fever of unknown origin and if the surgery is not performed for an emergency situation, the surgery should be postponed (9). In our patient group, the suspicious contact history of the pre-operative patients and their history of recent foreign trips were questioned, as well. Pre-operative fever check, chest x-ray, abdominal CT and thorax CT for staging were also evaluated for both metastasis and COVID-19 findings. In blood tests lymphocyte count, ferritin level, d-dimer level were evaluated in terms of possible COVID-19 infection. As a result of the evaluations mentionedabove, there were no suspicious symptoms for COVID-19 in our patients.

To prevent cross-infection in the operating room, it should be ensured that all anaesthetic equipment, tools, and medications must be used for only one patient. Anaesthetic devices that come into contact with the respiratory tract, such as video laryngoscope lenses, plastic breathing tubes, filters, breathing balloons, suction tubes and sputum suction tubes, should be discarded after a single use. It should be ensured that healthcare professionals use their protective equipment (10).

Various triage methods have been designed to evaluate the risk of virus exposure with the progression of surgical disease and to determine the priority of surgery; the methods have also been designed to define the procedures that can be postponed or should not be performed in patients who are planned for surgery. The triage related to the management of patients and their treatment during the pandemic period has been reported by the American College of Surgeons (ACS) and Ross and his colleagues.

The triage and management of the surgical patients during the pandemic are divided into three phases by ACS. Phase 1 is named as semi-emergency environment (preparation phase). There are few COVID-19 patients in this phase and hospital resources are not depleted. The intensive care ventilator capacity is available and sufficient; COVID-19 cases tend to rise rapidly. If the patients are not operated within the next three months, limited surgery is planned for patients likely to be at risk of survival. Phase 2 is named as an emergency environment. There are a large number of COVID-19 patients; the numbers of intensive care unit, ventilators and operating rooms are limited or there is a rapid increase in COVID-19 cases within the hospital. Surgical procedures are limited to patients whose health could be in danger if the surgery is not performed within few days. In Phase 3, all hospital resources are depleted for COVID-19, there are no intensive care units and ventilators. Operating room consumable materials are depleted, as well. In this phase, surgical procedures are limited to patients whose health could be in danger if they are not operated within a few hours (6).

In the management of surgical patients during the pandemic period, (created by Ross and his colleagues) there are no COVID-19 patients in the phase of pandemic alert. Normal surgical procedures are continued to be performed. The first COVID-19 patient was detected in the Stage 2 pandemic phase. Elective surgical cases should be reduced by $50 \%$ and surgical emergency cases should be a priority. High-risk cases should be avoided. In the Stage 1 pandemic phase, the capacity of the facility was exceeded $100 \%$ and the intensive care capacity was exceeded over $90 \%$. In this phase, all elective operations must be stopped. Only emergency and mandatory surgical procedures should be performed (11). The surgical procedures can be performed according to the evaluation of the underlying cause and the risk of viral spread of the cases that can be postponed up to four weeks during the COVID-19 pandemic. This category (phase) covers some conditions that do not respond to cancer surgery and medical treatment. ACS determined the stages of surgical procedures according to the importance and risk of the surgery (high, medium, low elective surgical acuity scale) and the general condition of the patient. Cancer cases constitute the majority of surgeries with high risk threshold and the surgeries should not be delayed during the COVID-19 pandemic (6).

According to the recommendations of ACS, the curative surgery should be performed for resectable colon cancer, neoadjuvant chemotherapy can be considered for locally advanced colon cancer cases. For rectal cancer cases, all options of neoadjuvant chemotherapy should be considered. Patients who do not respond to neoadjuvant chemotherapy should be given priority in surgery. In the case where all hospital resources are channelled to COVID-19 patients, surgery should be limited to emergencies (perforation, peritonitis, massive gastrointestinal bleeding, sepsis) considering the transfer of patients to the hospital with capacity in all other cases (6).

In contrast to the above recommendations of ACS, the authors (12), which consider that neoadjuvant chemotherapy increases the $\mathrm{R} 0$ resection rate in locally advanced colon cancer patients, suggest that neoadjuvant chemotherapy should be applied to the patients and the surgical treatment should be delayed during the COVID-19 pandemic (13). In the National Comprehensive Cancer Network (NCCN) guidelines, it is suggested that evaluation of the response to the neoadjuvant chemo-radiotherapy in rectal cancer patients must be carried out between 5 th and 12 th weeks. Studies that do not approve of surgery for colorectal cancer patients during the COVID-19 pandemic argue that the waiting period after neoadjuvant chemo-radiotherapy can be extended to 16-24 weeks during the COVID-19 pandemic (14).

As it can be understood from the studies mentioned-above, the approach to the colorectal cancer patients during the COVID-19 period is generally to give neoadjuvant treatments and postpone surgical treatment. However, the triage guidelines of ACS advocate that the surgeries of colorectal cancer patients should not be delayed. In our own clinic, we performed the surgical treatment of the patients with colorectal cancer during the 


\section{3-296}

COVID-19 pandemic. After carrying out above-mentioned procedures and eliminating the COVID-19 suspicions in our patients, we performed the operations. Treatments of these patients were carried out in the services and operating rooms that were physically separated from the services and operating rooms, which are reserved for COVID-19 patients. Both, in inpatient services and in the operating room, the medical personnel performed the operations and service treatments of COVID-19 (-) colorectal cancer patients with Grade 1 protection measures according to the three-grade occupational protection algorithm (13) recommended for clinicians during COVID-19 pandemic. No further problems occurred related to COVID-19 pandemic in the post-operative follow-up period of the operated colorectal cancer patients. All patients were discharged when they completed their recovery process after the operations.

\section{Conclusion}

As a result, colorectal cancer diseases can be treated curatively with surgical treatment. Delaying the surgical treatment in this patient group has a negative effect on the patient's disease-free survival time. According to the ACS pandemic triage categorization, the hospital in which our clinic was located had Phase 2 hospital conditions and according to the triage categorization of Ross and his colleagues, the hospital had Stage 2 hospital conditions. Under these circumstances, we have demonstrated with our study that during the COVID-19 pandemic, surgical treatment can be performed for the patients with colorectal cancer by eliminating COVID-19 suspicions.

\section{References}

1. Organization WH. Country \& technical guidance-Coronavirus disease (COVID-19). Geneva, Switzerland: WHO. 2020.

2. Chan JF-W, Yuan S, Kok K-H et al. A familial cluster of pneumonia associated with the 2019 novel coronavirus indicating person-to-person transmission: a study of a family cluster. The Lancet. 2020; 395: 514-523.

3. Tian Y, Rong L, Nian W, He Y. gastrointestinal features in COVID-19 and the possibility of faecal transmission. Alimentary pharmacology \& therapeutics. 2020; 51: 843-851.

4. Control CfD and Prevention. Interim guidance for healthcare facilities: preparing for community transmission of COVID-19 in the United States. Page last reviewed February. 2020; 29.

5. Organization WH. WHO Director-General's opening remarks at the media briefing on COVID-19-11 March 2020. 2020.

6. Moletta L, Pierobon ES, Capovilla G et al. International guidelines and recommendations for surgery during covid-19 pandemic: a systematic review. Internat J Surg 2020.

7. Bolin S, Nilsson E, Sjödahl R. Carcinoma of the colon and rectum-growth rate. Annals of surgery. 1983; 198: 151.

8. Elster E, Potter BK, Chung K. Response to COVID-19 by the surgical community. Surgery. 2020.

9. Zhao S, Ling K, Yan H et al. Anesthetic management of patients with suspected 2019 novel coronavirus infection during emergency procedures. Journal of cardiothoracic and vascular anesthesia. 2020.

10. Radonovich Jr L, Simberkoff M, Bessesen M. ResPECT investigators. N95 respirators vs medical masks for preventing influenza among health care personnel. JAMA. 2019; 322: 824-833.

11. Ross SW, Lauer CW, Miles WS et al. Maximizing the calm before the storm: tiered surgical response plan for novel coronavirus (COVID-19). J Amer Coll Surg 2020.

12. Dehal A, Graff-Baker AN, Vuong B et al. Neoadjuvant chemotherapy improves survival in patients with clinical T4b colon cancer. J Gastrointest Surg 2018; 22: 242-249.

13. Ren $\mathrm{X}$, Chen B, Hong $\mathrm{Y}$ et al. The challenges in colorectal cancer management during COVID-19 epidemic. Ann Translational Med 2020; 8.

14. Habr-Gama A, Sabbaga J, Gama-Rodrigues J et al. Watch and wait approach following extended neoadjuvant chemoradiation for distal rectal cancer: are we getting closer to anal cancer management? Dis Colon Rectum 2013; 56: 1109-1117. 